\title{
Wetting Behavior of Liquids on Micro-Pattemed Polymer Surfaces Fabricated by Thermal Imprinting
}

\author{
Kwang-Jin Bae ${ }^{1,2}$, Wenhui Yao ${ }^{1}$, Yiliang $\mathrm{He}^{2}$, and Young-Rae Cho ${ }^{1, *}$ \\ ${ }^{1}$ Department of Materials Science and Engineering, Pusan National University, Busan 46241, Republic of Korea \\ ${ }^{2}$ School of Environmental Science and Engineering, Shanghai Jiao Tong University, Shanghai 200240, China
}

\begin{abstract}
The wetting behavior of liquids on solid surfaces is important in the study of surfaces and interfaces. In this study, the wetting behavior of deionized (DI) water and hexadecane on fluorinated and micro-patterned polymer surfaces was investigated. The sample surfaces were processed by spray coating and thermal imprinting, respectively. The apparent contact angle and sliding angle of each sample were measured. Hexadecane, which has a low surface tension, spread easily over the micro-patterned surface, indicating oleophilicity. However, the apparent contact angle of DI water on the micro-patterned surface was approximately $134.2^{\circ}$, indicating that the surface was hydrophobic. Although the apparent contact angle of DI water on the micro-patterned surface indicated hydrophobicity, the sliding angle was very high at $60^{\circ}$. These results indicated that DI water droplets on the micro-patterned surfaces have an intermediate wetting state between the Wenzel and Cassie-Baxter states. Based on the experimental results, it was concluded that the wetting behavior of DI water droplets on the micro-patterned surfaces is determined by the force balance between the capillary force and the air counterforce.
\end{abstract}

(Received April 3, 2017; Accepted May 14, 2017)

Keywords: apparent contact angle, intermediate wetting state, micro-patterned surface, sliding angle, wetting behavior

\section{INTRODUCTION}

The characteristic known as wettability is one of the most important properties in the study of the wetting behavior of surfaces, and the bonding behavior of interfaces. Wettability has been widely studied in research on composite materials, solar cells, electronic devices, and antimicrobial films [1-5]. The wetting behavior of the surfaces of solid materials is affected by two factors: surface energy and surface roughness [6]. Surface energy can be controlled by chemical modification methods, such as fluorination. At the same time, surface roughness can be modified by etching, lithography, template-assisted synthesis, sputter deposition, galvanic replacement reaction and sol-gel synthesis [7-13].

Therefore there has been extensive research on the use of surface modifications to create special wetting properties on solid surfaces. For example, Wang and coworkers applied perfluorodecanol on multi-wall carbon nanotubes (MWCNTs)

*Corresponding Author: Young-Rae Cho [Tel: +82-51-510-2389, E-mail: yescho@pusan.ac.kr]

Copyright (c) The Korean Institute of Metals and Materials to synthesize fluorinated MWCNTs [14]. Yang and coworkers created hierarchical textured surfaces by hydrochloric acid $(\mathrm{HCl})$ etching [15]. Well-defined structures with micro-sized pillars and overhang re-entrant structures were produced by Zhao via a traditional photolithographic technique [16]. Fujii and coworkers prepared hierarchical pillar surfaces by sputtering of Al-Nb alloys onto aluminum substrates [17].

When liquid droplets fall onto rough surfaces, such as micro-patterned surfaces, the wetting behavior of the liquids can be explained by either the Wenzel model [18] or the Cassie-Baxter model [19]. In the Wenzel model, the droplet completely penetrates between the micro-patterns, while in the Cassie-Baxter model, air is trapped between these micro-patterns and the droplet stays on top of the micro-patterns and the trapped air [20]. In most research, the wetting behavior of liquids on solid surfaces is described by just using the static contact angle, which is commonly represented by the apparent contact angle [21]. In the case of rough surfaces, however, the wetting behavior of liquids 

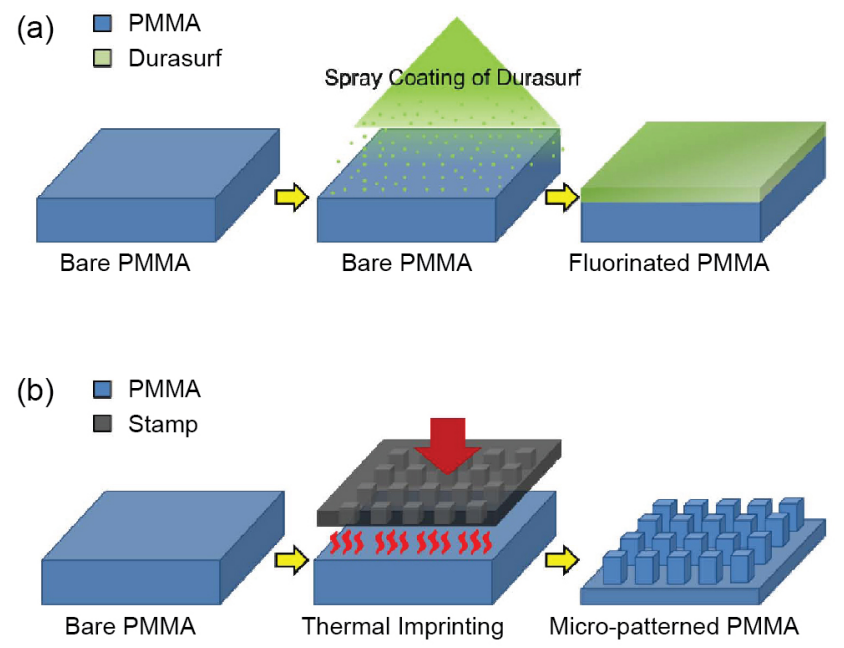

Fig. 1. Schematic diagram of the sample preparation: (a) fabrication of fluorinated polymethyl methacrylate (PMMA), and (b) thermal imprinting process to create micro pillars on the PMMA surface.

cannot be adequately described solely in terms of the apparent contact angle [22,23].

In this work, we measured the static contact angles of deionized (DI) water and hexadecane on fluorinated and micro-patterned polymer surfaces and compared them to those on bare polymer surfaces. A deeper understanding of the wetting behavior of liquids on surfaces can be obtained by also measuring the sliding angle of the DI water on the micro-patterned surface. For this reason, a combined study on wetting behavior using both apparent contact angle and sliding angle was carried out and analyzed, to obtain a better understanding of the wetting behavior of liquids on a micro-patterned surface.

\section{EXPERIMENTAL PROCEDURES}

\subsection{Preparation of samples}

Polymethyl methacrylate (PMMA) film (C.Y. Ltd., Korea) was selected as the substrate material because of its transparency, flexibility, low price and light weight. These are important properties for electronic and industrial applications. The thickness of the bare PMMA film was $175 \mu \mathrm{m}$ and the glass transition temperature of the PMMA was approximately $110{ }^{\circ} \mathrm{C}[24]$.
Table 1. Thermal imprinting conditions used in the PMMA micro-patterning process.

\begin{tabular}{cc}
\hline Parameter & Condition \\
\hline Materials & PMMA \\
Imprinting temperature & $150{ }^{\circ} \mathrm{C}$ \\
Pressure & $35 \mathrm{kN}$ \\
Imprinting time & $10 \mathrm{~min}$ \\
Cooling time & $30 \mathrm{~min}$ \\
\hline
\end{tabular}

Figure 1 shows a schematic diagram of the fabrication processes of the samples. Two types of sample, one with a fluorinated layer and one with a micro-patterned surface, were prepared by spraying and thermal imprinting processes, respectively. The sample with the fluorinated layer was produced by spraying a Durasurf solution (Harves. Co, Japan) onto a bare PMMA surface from a distance of $20 \sim 30 \mathrm{~cm}$ using an air brush (HP-CH; IWATA, Japan) with a nozzle diameter of $0.3 \mathrm{~mm}$ connected to a compressed air tank (pressure of 20 psi), as shown in Fig. 1(a).

The sample with a micro-patterned surface was manufactured by a thermal imprinting process, as shown in Fig. 1(b). Prior to fabricating the micro-patterned PMMA, a nickel (Ni) stamp containing the micro-patterns was prepared by photolithography and an electroforming process [25]. A negative photoresist (THB $126 \mathrm{~N}$; JSR. Co, Korea) was spin-coated on a silicon wafer, which was then baked at 110 ${ }^{\circ} \mathrm{C}$ to remove the excess solvent from the THB $126 \mathrm{~N}$ layer. The THB $126 \mathrm{~N}$ layer was exposed to ultraviolet (UV) light through a photomask (UV intensity $300 \mathrm{~mJ} / \mathrm{cm}^{2}$ ) for about $10 \mathrm{~s}$. After polymerization, the sample was developed in THB 126 $\mathrm{N}$ developer solution for $3 \mathrm{~min}$. Finally, the Ni electroforming process was carried out and the silicon was completely removed using potassium hydroxide $(\mathrm{KOH})$ solution. The thermal imprinting parameters used when fabricating the micro-patterned PMMA are listed in Table 1.

\subsection{Characterization of samples}

The wetting properties of DI water (surface tension: 72.7 dyne $/ \mathrm{cm}$ ) and hexadecane (surface tension: $27.6 \mathrm{dyne} / \mathrm{cm}$ ) on the fluorinated (F-PMMA), micro-patterned (M-PMMA) and micro-patterned PMMA with fluorinated (MF-PMMA) surfaces were investigated and compared to those of a bare 
Table 2. Notation and data of each sample for different fabrication methods.

\begin{tabular}{ccc}
\hline Notation & Condition & $\begin{array}{c}\text { Surface Energy } \\
(\text { dyne } / \mathrm{cm})\end{array}$ \\
\hline B-PMMA & Bare PMMA & 40.2 \\
F-PMMA & Fluorinated PMMA & 18.6 \\
M-PMMA & Micro-patterned PMMA & 40.2 \\
MF-PMMA & Micro-patterned PMMA with & 18.6 \\
\hline
\end{tabular}

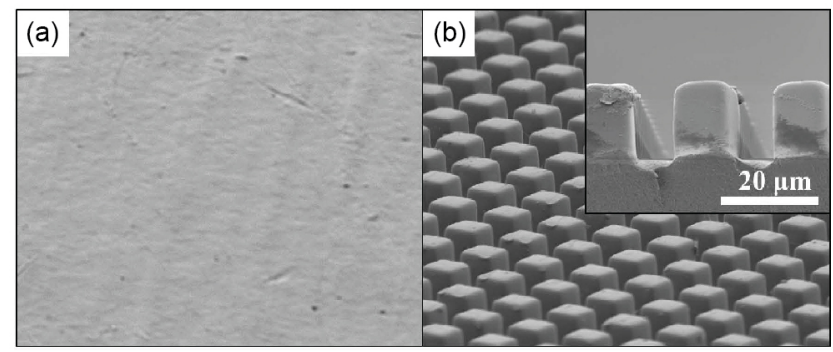

Fig. 2. Scanning electron microscope (SEM) images of bare PMMA and micro-patterned PMMA: (a) tilted view of the bare PMMA surface, and (b) tilted view of micro pillars on the PMMA surface. The inset shows an SEM image of the cross section for the micro-patterned PMMA.

PMMA (B-PMMA) surface $[6,26]$. The surface energy of each substrate was also measured using the Owens-Wendt model, based on the apparent contact angle of DI water and diiodomethane (surface tension: 50.8 dyne/cm) $[27,28]$. The surface morphology of the bare and micro-patterned PMMA surfaces was observed using scanning electron microscopy (SEM) (MIRA3; Tescan, Czech Republic). The apparent contact angles, which are a form of static contact angles, of DI water and hexadecane (ReagentPlus ${ }^{\circledR}$, 99\%; SigmaAldrich, Korea) on the B-PMMA, F-PMMA, M-PMMA and MF-PMMA surfaces were measured using a SmartDrop (FEMTOFAB Co., Korea). The sliding angles, which are a form of dynamic contact angles, of DI water on the B-PMMA, F-PMMA, M-PMMA and MF-PMMA surfaces were measured by tilting the stage until incipient motion of the droplet was detected using the SmartDrop [29].

\section{RESULTS AND DISCUSSION}

\subsection{Surface morphology and surface energy}

Figure 2 shows SEM images of the bare and
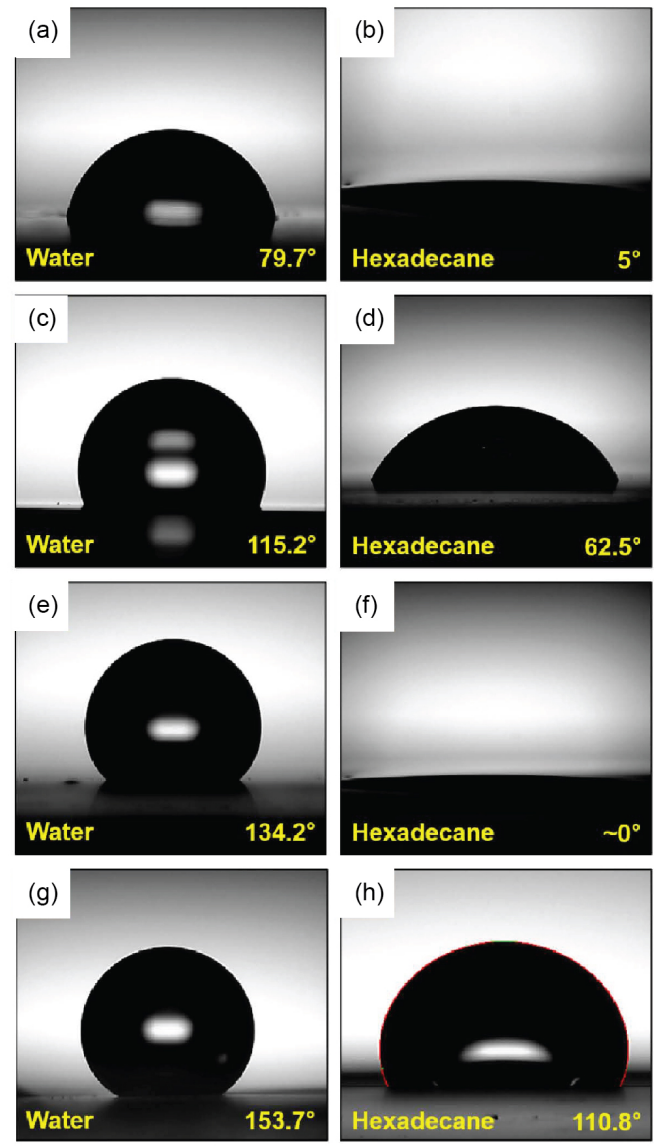

Fig. 3. Apparent contact angles (CAs) of the deionized (DI) water and hexadecane on the sample surfaces: (a) DI water droplet on bare PMMA, (b) hexadecane droplet on bare PMMA, (c) DI water droplet on fluorinated PMMA, (d) hexadecane droplet on fluorinated PMMA, (e) DI water droplet on micro-patterned PMMA, (f) hexadecane droplet on micro-patterned PMMA, (g) DI water droplet on micro-patterned PMMA with fluorination, and (h) hexadecane droplet on micro-patterned PMMA with fluorination.

micro-patterned PMMA surfaces. The surface of the bare PMMA sample shown in Fig. 2(a) is nearly flat. Micro-pillars are homogeneously distributed on the surface of the micro-patterned PMMA sample shown in Fig. 2(b). According to the inset image, the pitch and height of the micro-pillars on the surface of the PMMA sample were $20 \mu \mathrm{m}$ and $15 \mu \mathrm{m}$, respectively. The fluorinated layer was uniformly coated on the PMMA surface at a thickness less than $20 \mathrm{~nm}$. Accordingly, the fluorinated coating on the PMMA surface can be expected to be conformal. Table 2 shows the notations and surface energies of the bare PMMA, fluorinated PMMA, micro-patterned PMMA and micro-patterned PMMA with 
fluorination. The surface energy of each substrate was calculated from the apparent contact angle of DI water and diiodomethane using the Owens-Wendt model [27]. The surface energies of the bare and fluorinated PMMA were 40.2 dyne/cm and 18.6 dyne/cm, respectively. The surface energy of the micro-patterned PMMA was difficult to measure due to the unique surface structure, but it was expected to have the same value as the bare PMMA.

\subsection{Static contact angles}

The static contact angle was determined by measuring the apparent contact angle of a liquid droplet placed on the surface [21]. Figure 3 shows the apparent contact angles of the DI water and hexadecane for each sample. The apparent contact angles of the DI water and hexadecane on the bare PMMA surface were $79.5^{\circ}$ and $5^{\circ}$, respectively, as shown in Fig. 3(a) and (b). Both apparent contact angles on the bare PMMA surface were less than $90^{\circ}$, indicating that the bare PMMA surface was intrinsically hydrophilic and oleophilic $[6,7]$. In the case of the fluorinated PMMA surface shown in Fig. 3(c) and (d), the apparent contact angles of the DI water and hexadecane were $115.2^{\circ}$ and $62.5^{\circ}$, respectively. These are larger than the apparent contact angles of the liquids with the bare PMMA surface. In particular, the apparent contact angle of DI water on the micro-patterned PMMA surface was $134.2^{\circ}$, which is much higher than that on the bare PMMA, as shown in Fig. 3(e). On the other hand, the hexadecane droplet spread completely over the micro-patterned surface, with an apparent contact angle of $\sim 0^{\circ}$, as shown in Fig. 3(f). In case of the MF-PMMA surface, as shown in Figs. 3(g) and (h), the apparent contact angles of DI water and hexadecane were $153.7^{\circ}$ and $110.8^{\circ}$, which indicate super-hydrophobicity and oleophobicity, respectively.

Figure 4 shows the change in the apparent contact angles for the different liquids and substrates. The apparent contact angles of the DI water and hexadecane on the F-PMMA surface were higher than on the bare surface. This phenomenon may be explained by a decrease in surface energy. The comparison of the bare and fluorinated PMMA in Table 2 shows that the fluorine coating affects the surface energy, which decreased from $40.2 \mathrm{dyne} / \mathrm{cm}$ to $18.6 \mathrm{dyne} / \mathrm{cm}$.

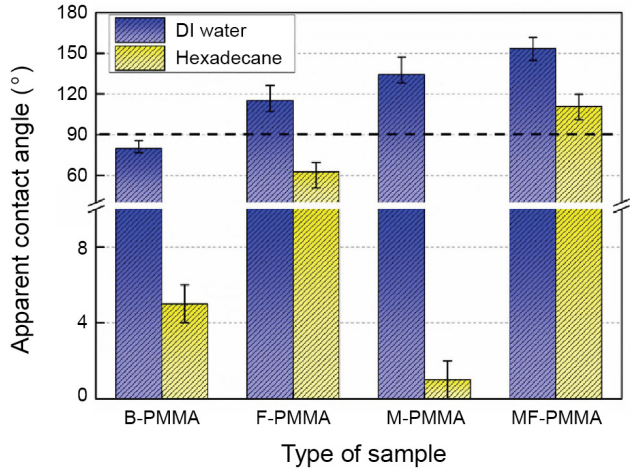

Fig. 4. Change in apparent contact angles of the DI water (blue bar) and hexadecane (yellow bar) on the bare PMMA (B-PMMA), fluorinated PMMA (F-PMMA), micro-patterned PMMA (M-PMMA), and micro-patterned PMMA with fluorination (MF-PMMA).

Therefore, the increase in the apparent contact angles was attributed to the decrease in surface energy, which indicates low adhesion at the solid-liquid interface [30]. For the same reason, the apparent contact angles of the DI water and hexadecane on the MF-PMMA surface were also higher than on the M-PMMA surface. This result explains why fluorine coatings are widely used in liquid-repellent surfaces [31].

The DI water and hexadecane exhibited different wetting behaviors when they were in contact with the micro-patterned surface. The micro-patterned surface was not flat; therefore, the wettability was affected by the surface roughness. In the Wenzel model, a liquid droplet is in perfect contact with the surface due to its strong capillarity, and there is no trapped air below the liquid droplet. In the Cassie model, the liquid droplet is placed on top of the micro-patterned surface, and the trapped air prevents the liquid from penetrating into the micro-patterns [32].

The contact angle of the liquid droplet in the Wenzel model can be expressed by the following equation [18]:

$\cos \theta_{\mathrm{w}}=\mathrm{r} \cos \theta_{\mathrm{i}}$

where $\theta_{\mathrm{w}}$ is the apparent contact angle on the micro-patterned surface, $r$ is the surface roughness factor $(r>1)$, and $\theta_{i}$ is the intrinsic contact angle on a flat surface of the same material. When the intrinsic contact angle $\theta_{i}$ is less than $90^{\circ}$, the apparent contact angle $\theta_{\mathrm{w}}$ will be decreased by a surface 
Table 3. Wettability related data for samples obtained from the deionized water.

\begin{tabular}{cccccc}
\hline Notation & $\begin{array}{c}\text { Contact } \\
\text { angle } \\
\text { (deg) }\end{array}$ & $\begin{array}{c}\text { Sliding } \\
\text { angle } \\
(\mathrm{deg})\end{array}$ & $\begin{array}{c}\text { Advancing Receding } \\
\text { angle } \\
(\mathrm{deg})\end{array}$ & $\begin{array}{c}\text { angle } \\
\text { (deg) }\end{array}$ & $\begin{array}{c}\text { Hysteresis } \\
(\mathrm{deg})\end{array}$ \\
\hline B-PMMA & 79.7 & 74 & 80.2 & 65.2 & 15 \\
F-PMMA & 115.2 & 65 & 132.7 & 105.4 & 27.3 \\
M-PMMA & 134.2 & 60 & 149.3 & 114.2 & 35.1 \\
MF-PMMA & 153.7 & 42 & 150 & 113.3 & 36.7 \\
\hline
\end{tabular}

roughness factor. Since bare PMMA surfaces are intrinsically oleophilic, the decrease in the hexadecane contact angle on the micro-patterned surface can be explained by the Wenzel model.

In contrast, although the bare PMMA surface was intrinsically hydrophilic, the contact angle of the DI water on the micro-patterned surface increased. This result indicates that DI water on a micro-patterned surface is characterized by the Cassie-Baxter model rather than the Wenzel model. The wetting behavior of the Cassie-Baxter state is governed by the following equation [19]:

$$
\cos \theta_{\mathrm{c}}=\varphi_{\mathrm{s}}\left(1+\cos \theta_{\mathrm{i}}\right)-1
$$

where $\theta_{\mathrm{c}}$ is the apparent contact angle on the textured (micro-pattered) surface, $\varphi_{\mathrm{s}}$ is the fraction of the solid in contact with the liquid, which is normally less than unity, and $\theta_{\mathrm{i}}$ is the intrinsic contact angle on a flat surface of the same material. In contrast to the Wenzel model, the apparent contact angle, $\theta_{c}$ increases with the surface roughness regardless of the intrinsic contact angle. However, in many cases, when a droplet is placed on a micro-patterned surface, the liquid partially enters the patterns [32]. This wetting model is called an intermediate wetting model, and has been observed by Luo et al. [33]. To understand the exact wetting behavior of the liquids on the micro-patterned surface, it is necessary to consider the sliding angle.

\subsection{Dynamic contact angles}

The dynamic behavior of liquids on surfaces can be characterized by the sliding angle and contact angle hysteresis. The sliding angle is defined as the critical inclination angle at which a droplet rolls down the surface
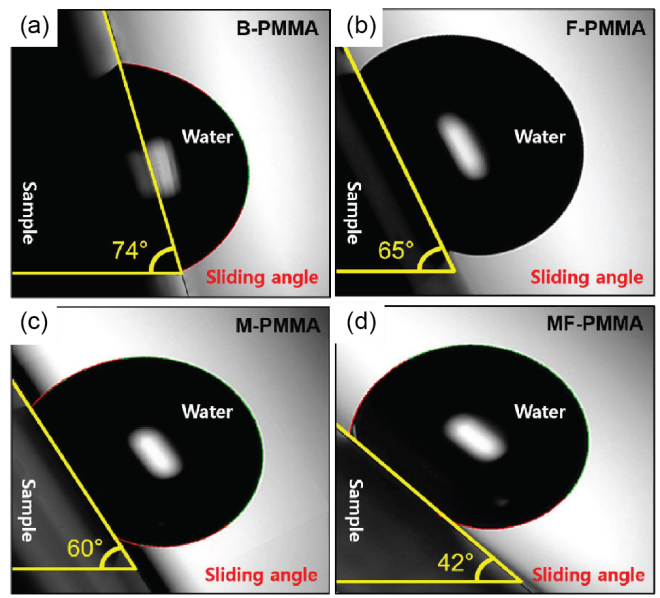

Fig. 5. Optical images of the sliding angle of DI water on the bare PMMA, fluorinated PMMA, micro-patterned PMMA and micro-patterned PMMA with fluorination surface: (a) sliding angle of DI water on the B-PMMA surface, (b) sliding angle of DI water on the F-PMMA surface, (c) sliding angle of DI water on the M-PMMA surface, and (d) sliding angle of DI water on the MF-PMMA surface.

[23]. The contact angle hysteresis is defined as the difference between advancing and receding angles when the tilting angle is changed [21].

Table 3 shows the data for the measured static and dynamic contact angles of DI water. It can be seen that the sliding angle is gradually decreased by fluorinating and micro-patterning. However, in contrast to the sliding angles, the contact angle hysteresis increased. This is because a water droplet on a micro-patterned surface has a smaller contact area, compared to a flat surface.

Figure 5 shows optical images of the sliding angle of DI water on the B-PMMA, F-PMMA, M-PMMA and MF-PMMA surfaces. As shown in Fig. 5(a) and (b), the sliding angles of DI water on the bare and fluorinated PMMA surface are about $74^{\circ}$ and $65^{\circ}$, indicating the sticky behavior of Wenzel model. Because of the micro pattern on the M-PMMA and MF-PMMA surfaces, the water droplets were expected to exhibit the non-sticky behavior of the Cassie-Baxter model. However, the values of the sliding angle were found to be about $60^{\circ}$ and $42^{\circ}$, indicating a sticky surface, as shown in Fig. 5(c) and (d). From the comparison of Fig. 5(c) and (d), it can be concluded that the fluorinated coating decreased the sliding angle by reducing the surface 


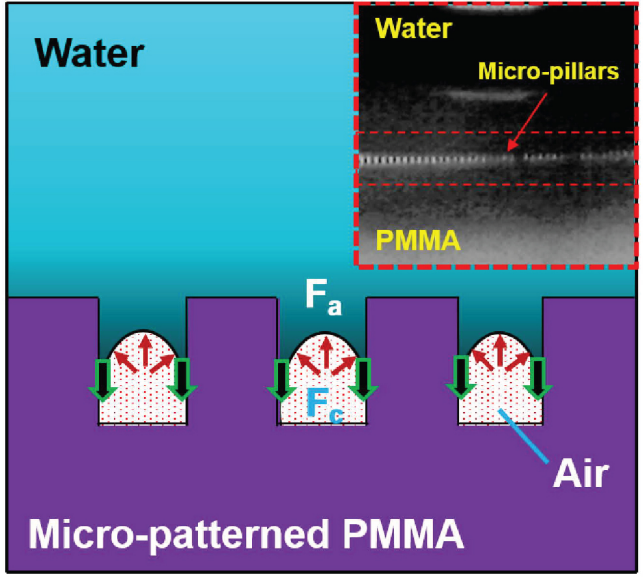

Fig. 6. Schematic diagrams of the solid-liquid interface on the micro-patterned PMMA surface. $F_{c}$ and $F_{a}$ represent capillary force and air counterforce, respectively. The inset shows an optical image of solid-liquid interface on the micro-patterned PMMA.

energy.

Theoretically, in the Cassie-Baxter model, a water droplet on a rough surface is described as non-sticky [21]. This means that the adhesion between the droplet and the surface is extremely low. Therefore, the droplet can easily roll down the surface when it is tilted slightly, and the sliding angle and contact angle hysteresis of the droplet on the surface is typically less than $10^{\circ}$ [7].

In this work, however, an abnormal phenomenon was observed: though the apparent contact angle of DI water on the micro-patterned PMMA surface was $134.2^{\circ}$ (Fig. 3(e)) indicating the hydrophobicity of the Cassie-Baxter model, the sliding angle of DI water on the micro-patterned PMMA surfaces was $60^{\circ}$, which is a kind of sticky behavior, indicating the hydrophilicity of the Wenzel model, as shown in Fig. 5(c). This indicates that DI water droplets on micro-patterned surfaces cannot be simply described by the Cassie-Baxter model alone, but are better described by the intermediate wetting model, between the Wenzel and the Cassie-Baxter models. Accordingly, the wetting behavior of DI water droplets on the micro-patterned surface should be appropriately investigated using a schematic diagram of the solid-liquid interface of the B-PMMA and M-PMMA surfaces.

Figure 6 shows a schematic diagram of the solid-liquid interface of the micro-patterned surfaces to explain the abnormal wetting behavior of the M-PMMA surface. In the inset image, a number of trapped air bubbles can be observed along the interface between the DI water droplets and the surface of the micro-patterns. Using the intermediate wetting model, both the hydrophobic and the sticky behaviors of the DI water droplets on the micro-patterned surfaces were considered. The intermediate wetting model can be explained as a competition between the capillary force and the counter force of the trapped air [32,34]. For the M-PMMA and MF-PMMA samples, as shown in Fig. 6, the capillary force $\left(F_{c}\right)$ acts at the interfaces between the liquid droplet and the sidewall of the micro-patterns. The air counterforce $\left(F_{a}\right)$ acts at the interface of the liquid droplet and the air trapped between the micro-patterns [35].

DI water droplets have a large surface tension of 72.7 dyne/cm, so the counterforce induced by the trapped air was stronger than the capillary force at the PMMA-DI water interface. Therefore, the DI water droplets did not fall into the gaps, but acted as bridges between the micro-pillars, and hence had a larger apparent contact angle. At the same time, unlike the Cassie-Baxter model, the trapped air could not fully counter the capillary force, as shown in Fig. 6. For the MF-PMMA sample, the capillary force between the liquid droplets and the micro-pillars decreased due to the reduced surface energy, which is related to the smaller sliding angle. Based on this result, it was concluded that the DI water partially penetrated the gaps between the patterned pillars on the PMMA surface. This behavior is described by the intermediate wetting model [36].

The wetting behavior of DI water on micro-patterned PMMA surfaces could not be explained rigorously by the Wenzel model or the Cassie-Baxter model, which only consider apparent contact angles. To accurately predict the wetting behavior of liquids on the micro-patterned surfaces, the intermediate wetting model, which requires a sliding angle, must be taken into account. Furthermore, to fabricate the perfect non-sticky surface of the Cassie-Baxter model, chemical surface modification is needed to produce low capillary forces, and micro-pillars with a small area fraction are required for large air counterforce [10]. 


\section{CONCLUSIONS}

Three types of sample were fabricated by spraying and thermal imprinting on PMMA surfaces, and then their wetting behavior was investigated. Although bare PMMA surfaces were hydrophilic, the apparent contact angle of the DI water on the micro-patterned PMMA surface increased to $134.2^{\circ}$, indicating hydrophobicity. Unexpectedly, the sliding angle of the DI water on the micro-patterned PMMA surface was very high at $60^{\circ}$. The abnormal phenomena of both the large apparent contact angle of $134.2^{\circ}$ and the unusually large sliding angle of $60^{\circ}$ for DI water on the micro-patterned PMMA surface could be rigorously explained using an intermediate wetting model. To fully predict the wetting behavior of liquids on the micro-patterned surfaces, the intermediate wetting model, which incorporates the sliding angle, must be taken into account. For the fabrication of perfect non-sticky surface on the micro-patterned PMMA, chemical surface modification is needed to produce low capillary force, and an optimized structural design is required to produce a large air counterforce.

\section{ACKNOWLEDGEMENT}

This research was supported by the Basic Science Research Program of the National Research Foundation of Korea (NRF), which is funded by the Ministry of Education (2016R1D1A1B02009234). This work was also supported by "Human Resources Program in Energy Technology" of the Korea Institute of Energy Technology Evaluation and Planning (KETEP), granted financial resource from the Ministry of Trade, Industry \& Energy, Republic of Korea. (No. 20164010201000)

\section{REFERENCES}

1. Y. K. Jeong, I. G. Kim, and B. K. Ryu, Korean J. Met. Mater. 54, 461 (2016).

2. S. Kim, U.T. Jung, S. K. Kim, J. H. Lee, H. S. Choi, C. S. Kim, and M. Y. Jeong, ACS Appl. Mater. Inter. 7, 326 (2015).

3. J. Zhu, C. M. Hsu, Z. Yu, S. Fan, and Y. Cui, Nano Lett.
10, 1979 (2010)

4. H. J. Park, Y. S. Kim, J. H. Kim, I. G. Kim. J. Y. Jung. S. Y. Choi, and B. K. Ryu, Korean J. Met. Mater. 53, 237 (2015).

5. Y. K. Jeong, I. G. Kim, and B. K. Ryu, Korean J. Met. Mater. 54, 461 (2016).

6. A. Tuteja, W. Choi, M. Ma, J. M. Mabry, S. A. Mazzella, G. C. Rutledge, G. H. McKinley, and R. E. Cohen, Science 318, 1618 (2007).

7. Z. Chu and S. Seeger, Chem. Soc. Rev. 43, 2784 (2014).

8. K. Ellinas, S. P. Pujari, D.A. Dragatogiannis, C. A. Charitidis, A. Tserepi, H. Zuilhof, and E. Gogolides, ACS Appl. Mater. Inter. 6, 6510 (2014).

9. H. Zhao, K. C. Park, and K. Y. Law, Langmuir 28, 14925 (2012).

10. T. Li, M. Paliy, X. Wang, B. Kobe, W. M. Lau, and J. Yang, ACS Appl. Mater. Interfaces 7, 10988 (2015).

11. H. Ennaceri, L. Wang, D. Erfurt, W. Riedel, G. Mangalgiri, A. Khaldoun, A. E. Kenz, A. Benyoussef, and A. Ennaoui, Surf. Coat. Tech. 299, 169 (2016).

12. N. V. Phuong, B. R. Fazal, and S. Moon, Met. Mater. Int. 23, 106 (2017).

13. B. C. Park and Y. K. Kim, Met. Mater. Int. 23, 413 (2017).

14. X. Wang, H. Hu, Q. Ye, T. Gao, F. Zhou, and Q. Xue, J. Mater. Chem. 22, 9624 (2012).

15. J. Yang, Z. Zhang, X. Xu, X. Men, X. Zhu, and X. Zhou, New J. Chem. 35, 2422 (2011).

16. H. Zhao, K. Y. Law, and V. Sambhy, Langmuir 27, 5927 (2011).

17. T. Fujii, Y. Aoki, and H. Habazaki, Langmuir 27, 11752 (2011).

18. R. N. Wenzel, Ind. Eng. Chem. 28, 988 (1936).

19. A. B. D. Cassie and S. Baxter, Trans. Faraday Soc. 40, 546 (1944).

20. C. Luo, M. Xiang, X. Liu, and H. Wang, Microfluid. Nanofluid. 10, 831 (2011)

21. H. Bellanger, T. Darmanin, E. T. Givenchy, and F. Guittard, Chem. Rev. 114, 2694 (2014).

22. J. H. Song, M. Sakai, N. Yoshida. S. Suzuki, Y. Kameshima, and A. Nakajima, Surf. Sci. 600, 2711 (2006).

23. X. Li, L. Zhang, X. Ma, and H. Zhang, Surf. Coat. Technol. 307, 243 (2016).

24. C. E. Porter and F. D. Blum, Macromolecules 33, 7016 (2000).

25. H. S. Lee, H. H. Yang, S. Ra, and J. B. Yoon, J. Micromech. Microeng. 21, 065026 (2011).

26. J. Drelich and J. D. Miller, Ann. Univ. M. Curie-Sklodowska. AA. Chem. 54, pp. 105-115 (1999).

27. D. K. Owens and R. C. J. Wendt, J. Appl. Polym. Sci. 13, 1741 (1969).

28. B. Janczuk, W. Wojcik, and A. Zdziennicka, J. Colloid. Interf. Sci. 157, 384 (1993).

29. D. Oener and T. J. McCarthy, Langmuir 16, 7777 (2000). 30. W. A. Zisman, Advances in Chemisrty Series 43, pp. 
1-51 (1963).

31. D. Ge, L. Yang, Y. Zhang, Y. Rahmawan, and S. Yang, Part. Part. Syst. Charact. 31, 763 (2014).

32. J. Yang, J. Wang, C. W. Wang, X. He, Y. Li, J. B. Chen, and F. Zhou, Thin Solid Films 562, 353 (2014).

33. C. Luo, M. Xiang, and X. Heng, Langmuir 28, 9554 (2012).
34. Z. Li, J. Wang, Y. Zhang, J. Wang, L. Jiang, and Y. Song, Appl. Phys. Lett. 97, 233107 (2010).

35. C. Ran, G. Ding, W. Liu, Y. Deng, and W. Hou, Langmuir 24, 9952 (2008).

36. J. Li, Z. Jing, Y. Yang, Q. Wang, and Z. Lei, Surf. Coat. Tech. 258, 973 (2014). 